Cite this: J. Mater. Chem. A, 2013, 1, 4431

Received 22nd January 2013

Accepted 19th February 2013

DOI: $10.1039 /$ c3ta10337d

www.rsc.org/MaterialsA

\section{Plasma-assisted simultaneous reduction and nitrogen doping of graphene oxide nanosheets}

\author{
Nanjundan Ashok Kumar, $\ddagger^{\mathrm{a}}$ Hugo Nolan, $\ddagger^{\mathrm{ab}}$ Niall McEvoy, $\ddagger^{\mathrm{a}}$ Ehsan Rezvani, ${ }^{\text {ab }}$ \\ Richard L. Doyle, ${ }^{b}$ Michael E. G. Lyons ${ }^{b}$ and Georg S. Duesberg*ab
}

\begin{abstract}
An environmentally benign and scalable route for the production of gram scale quantities of nitrogen-doped graphene using a downstream microwave plasma source is reported. Simultaneous reduction and doping of graphene oxide is achieved and the process negates the need for high temperatures and toxic solvents associated with existing methods. This gas-phase low temperature process is completely dry and, thus, minimises re-aggregation of graphene flakes which is typically associated with liquid phase reduction methods. The resulting material has many potential uses, particularly in electrochemical energy.
\end{abstract}

Recent research has shown that graphene based materials can be tuned by chemical or structural modification; engendering them with properties more suited to specific applications than pristine graphene. In particular, nitrogen doped (N-doped) graphene has been extensively studied as it is an intriguing nanomaterial with tremendous potential for a myriad of applications ranging from energy to sensing. ${ }^{1,2}$ It is widely accepted that doping heteroatoms into the graphitic lattice can tailor its chemical and physical properties. N-doped carbon nanostructures show n-type or metallic behaviour and are expected to display greater electron mobility whilst introducing chemically active sites for use in electrocatalytic reactions; which are of great significance in fuel cells, ${ }^{3}$ lithium ion batteries ${ }^{4}$ and sensing. ${ }^{5}$ Although their fascinating properties endow them with great potential, the successful adoption of graphene based materials for real time applications largely relies on factors such as cost, processability and mass production.

Considerable effort has been devoted to developing preparation methods for high quality graphene including chemical

\footnotetext{
${ }^{a}$ Centre for Research on Adaptive Nanostructures and Nanodevices (CRANN), Trinity College Dublin, Ireland

${ }^{b}$ School of Chemistry, Trinity College Dublin, Ireland. E-mail: duesberg@tcd.ie; Fax: +35318963037

† Electronic supplementary information (ESI) available: Synthesis and experimental details are presented. See DOI: 10.1039/c3ta10337d

$\ddagger$ These authors contributed equally to this work.
}

vapour deposition (CVD) and solution based synthetic routes. ${ }^{6}$ Among various synthetic methods, chemical graphitisation of graphene oxide (GO) to its reduced form remains an established method for mass production at relatively low cost. ${ }^{7}$ Hitherto, the reduction process itself is usually fulfilled by chemical methods which rely heavily on different toxic reductants. Reduction of GO can also be achieved through thermochemical, ${ }^{8}$ solvothermal and hydrothermal methods; ${ }^{9}$ however, these processes stand inadequate for the mass production of graphene and most of the reduction mechanisms reported to date remain ambiguous. Similar methods stand valid regarding preparation of $\mathrm{N}$-doped graphene; of which, thermal annealing, ${ }^{10}$ arc discharge ${ }^{11}$ and CVD $^{12}$ have frequently been used. Unfortunately, the aforementioned strategies do not always give the required level of doping and often the poor yield or scalability limits the practical application of graphene obtained using such techniques. In addition, the high temperatures typically required for doping lead to poor processing and high energy consumption. Elevated temperatures may also lead to the removal of pyridinic nitrogen groups, ${ }^{\mathbf{1 0}}$ thought to be important for certain electrochemical applications, in particular oxygen reduction catalysis. ${ }^{13-15}$ Thus, the development of low temperature production of $\mathrm{N}$-doped graphene may be considered advantageous.

Plasma treatments provide one promising option to circumvent this problem, as an eco-friendly and energy-saving alternative to existing chemical processes. Recently, Wang et al. ${ }^{5}$ reported the preparation of $\mathrm{N}$-doped graphene by plasma treatment which resulted in a doping level of up to $1.35 \%$. In another study, N-doped graphene was prepared by dispersing GO in ethanol and coating this onto glassy carbon followed by exposure to $\mathrm{N}_{2}$ plasma. ${ }^{2}$ In another instance, GO powders were first thermally exfoliated and then subjected to $\mathrm{N}_{2}$ plasma to obtain a doping level of $3 \% .^{16}$ Though doping is achieved to a certain level, the $\mathrm{N}$-doped graphene materials obtained after subjection to elevated temperatures or by liquid-phase, in general, tend to re-aggregate thus hampering their potential. For applications that require a large graphene inter-sheet 
distance, such as battery anodes and supercapacitor electrodes, this aggregation factor remains a hindrance, making room temperature processing preferable. Although a variety of techniques have been reported to dope graphene with heteroatoms, until today, only a handful of studies report the simultaneous reduction and doping of graphene. ${ }^{1}$ Consequently, methodologies for simultaneous reduction and doping of GO need to be developed for graphene based materials to realise their potential for various applications. A persistent challenge surrounding these methodologies, however, is achieving a high level of doping and controlled reduction with minimal aggregation.

Herein, we report a facile approach to simultaneously reduce and nitrogen-dope GO near room temperature by employing a plasma-assisted downstream microwave technique. This process negates the need for high temperatures and toxic solvents associated with existing methods. Furthermore, bulk quantities of graphene sheets doped with a high level of nitrogen atoms can be achieved where $\mathrm{N}$-atoms are substitutionally doped into the graphene lattice; which is not readily realised by most other methods. This reproducible, green, one-pot process is completely dry and, thus, eliminates the re-aggregation of graphene flakes.

Graphene used in the current work was prepared by a chemical method based on the Hummers and Offeman oxidation as reported previously. ${ }^{17}$ Plasma treatments were carried out in a $\mathrm{R}^{3} \mathrm{~T}$ TWR-2000T microwave plasma generator. Gram scale quantities of GO were placed inside the chamber and exposed to a gas mixture of $\mathrm{H}_{2}$ and $\mathrm{NH}_{3}(50 \mathrm{sccm}$ each) at a power of $500 \mathrm{~W}$ for a dwell time of 1 hour. Temperature control was achieved through active water cooling of the walls of the plasma chamber, ensuring that the sample did not significantly heat. The downstream nature of the plasma means that the microwave generator is remote from the GO sample itself; no direct heating of the material through microwave irradiation takes place. Internal temperature measurements of the reactor chamber in the vicinity of the sample indicate that the temperature only rises by approximately $10{ }^{\circ} \mathrm{C}$ over the course of a typical plasma treatment. X-ray photoelectron spectroscopy (XPS) measurements were carried out to probe the level of nitrogen atoms introduced into the graphitic lattice following plasma treatment.

Survey scans of the two materials show differences in their respective spectra, illustrating the contributions of different atomic species. The intensity of the $\mathrm{O} 1 \mathrm{~s}$ peak relative to the $\mathrm{C} 1 \mathrm{~s}$ peak is reduced in $\mathrm{N}$-doped graphene $(\mathrm{C} / \mathrm{O}$ ratio $=5.2)$ compared to $\mathrm{GO}(\mathrm{C} / \mathrm{O}$ ratio $=2.2)$. Also present in the $\mathrm{N}$-doped graphene is the characteristic N1s peak, which is absent in GO. The In $3 \mathrm{~d}$ peaks visible in the GO survey scan are contributions from the sample mounting procedure and can be ignored. The nitrogen introduced during the plasma treatment was found to comprise 5.8 at.\% of the material. The well-exfoliated nature of the material suggests that minimal quantities of the material are inaccessible to the plasma treatment; signifying that the surface sensitive XPS is a reasonable means for measuring the level of functionalisation. In any case, proposed applications for this material such as sensors and energy conversion and storage rely on surface processes. It is sufficient to probe the nature of the functionalisation through surface sensitive techniques when considering the material for these applications.
Analysis of the core level characteristic peaks allowed insight to be gained into the nature of the chemical bonds in each sample. Shown in Fig. 1 are high resolution spectra in the region of the C1s characteristic peak for GO (Fig. 1a) and $\mathrm{N}$-doped graphene (Fig. 1b). These are resolved into contributions from various carbon-oxygen binding arrangements. It should be noted that charge compensation was required for the insulating GO sample as the sample gained charge with emission of photoelectrons. This process often over compensates energy shifts due to charging and, in fact, causes the characteristic peaks to shift to lower binding energies than expected. This is seen in Fig. 1a where the GO C1s is observed to have downshifted from its expected position by about $1 \mathrm{eV}$.

The contributions from carbon-oxygen species were found to have been significantly reduced for the plasma treated sample when compared to the initial GO. The peak corresponding to graphitic, or $\mathrm{sp}^{2}$, carbon at $284.5 \mathrm{eV}$ is the major feature of the C1s region. ${ }^{18-20}$ The intensity of the $\mathrm{sp}^{3}$ carbon peak found at $285.5 \mathrm{eV},{ }^{20}$ is considerably reduced after plasma treatment; indicative of significant restoration of the graphitic lattice. Indeed, in GO this peak contribution is, in fact, greater than that of $\mathrm{sp}^{2}$ carbon; signifying the disruption to the initial graphitic lattice. The prominent doublet shape in the GO C1s region is due to the spectral contributions from oxygen functionalities at higher binding energies. Hydroxyl groups are seen as a peak at $\sim 286.7 \mathrm{eV},{ }^{\mathbf{1 , 2 1 , 2 2}}$ carbonyl groups are manifested at $\sim 288.2 \mathrm{eV},{ }^{\mathbf{1 , 2 0}}$ and carboxyl contributions appear at $\sim 289.3 \mathrm{eV}$. $^{5}$ The intensities of these peaks compared to the $\mathrm{C}=\mathrm{C}$ peak at $284.5 \mathrm{eV}$ are significantly reduced after plasma treatment; the doublet shape of the GO C1s region is changed to a single prominent peak with a shoulder in the N-doped graphene C1s region. As well as the reduction in oxygen moiety content, two further peaks are observed in the shoulder of this spectral region. These are attributed to carbon nitrogen bonds in $\mathrm{sp}^{2}$ $(\sim 286.1 \mathrm{eV})$ and $\mathrm{sp}^{3}$ configurations $(\sim 287.3 \mathrm{eV}) \cdot{ }^{21,23}$ Fitting of spectral contributions to the N1s region shows the composition
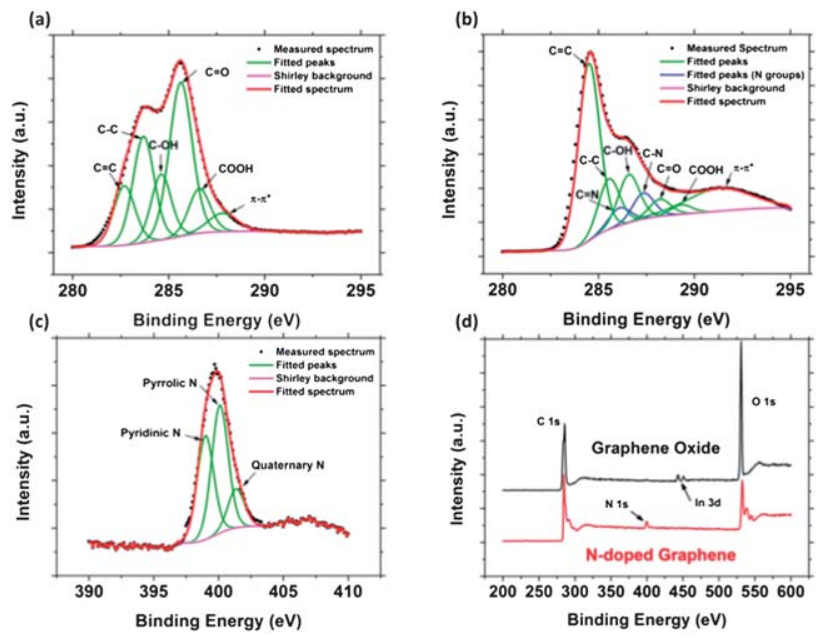

Fig. 1 XPS $C_{1 s}$ spectra of (a) GO and (b) reduced and N-doped graphene. (c) De-convoluted N1s spectrum showing contributions from pyridinic, pyrrolic and quaternary nitrogen species, (d) survey spectra of GO and N-doped graphene. 
of these nitrogen moieties in greater detail. Fig. 1c shows that the nitrogen groups present in the $\mathrm{N}$-doped graphene sample can be said to be comprised of pyridinic (peak at $\sim 399 \mathrm{eV})^{\mathbf{2 , 4 , 5}}$ pyrrolic (peak at $\sim 400.1 \mathrm{eV})^{23}$ and quaternary or graphitic nitrogen (peak at $\sim 401.3 \mathrm{eV})^{5,10,23}$ Some works attribute contributions in the region of the pyridinic or pyrrolic peaks to the presence of amine groups. ${ }^{\mathbf{1 0 , 2 3}}$

The reduction of the oxygen-containing groups in GO was also confirmed by FT-IR spectroscopy. As shown in Fig. 2a, it is evident that there are abundant oxygen containing groups, mainly carbonyl $(\mathrm{C}=\mathrm{O})$, present in GO. After reduction of the GO in plasma, however, the intensities of the FT-IR peaks corresponding to the oxygen functionalities, such as the $\mathrm{C}=\mathrm{O}$ stretching vibration peak at $1726 \mathrm{~cm}^{-1}$, decreased dramatically. These observations, taken together with the XPS data, confirm that oxygen functionalities in the GO were removed to some extent. Raman spectra, as shown in Fig. 2b, display typical D and $\mathrm{G}$ bands associated with graphitic materials. The $\mathrm{D}$ band $\left(\sim 1330 \mathrm{~cm}^{-1}\right)$ is a defect induced breathing mode of $A_{1 \mathrm{~g}}$ symmetry whereas the $\mathrm{G}$ band $\left(\sim 1580 \mathrm{~cm}^{-1}\right)$ stems from inplane vibrations and is of $E_{2 \mathrm{~g}}$ symmetry. The intensity ratio of the $\mathrm{D}$ to $\mathrm{G}$ bands $\left(I_{\mathrm{D}} / I_{\mathrm{G}}\right)$ is often used as a measure of defect levels in graphitic systems. Representative spectra of GO and $\mathrm{N}$-doped graphene are shown in Fig. 2b. It is observed that $I_{\mathrm{D}} / I_{\mathrm{G}}$ actually increases following plasma treatment $\left(I_{\mathrm{D}} / I_{\mathrm{G}}=0.97\right.$ before plasma treatment and 1.05 after). Initially this seems counterintuitive as one would expect lower defect levels following plasma treatment. Previously, authors have linked this increased $I_{\mathrm{D}} / I_{\mathrm{G}}$ with a smaller average crystallite size due to small crystals forming upon reduction. ${ }^{19}$ This observation can also be explained by considering the contribution of a second defect-induced band. The $\mathrm{D}^{\prime}$ band occurs via an intra-valley double resonance process in the presence of defects and is seen at $\sim 1610 \mathrm{~cm}^{-1}$. In highly defective graphitic systems this merges with the $G$ band. As defect levels are reduced, the intensity of the $\mathrm{D}^{\prime}$ band is decreased, which in turn makes the apparent observed $\mathrm{G}$ band intensity smaller. This is manifested in the downshifting observed in the $\mathrm{G}$ band following plasma treatment ( $\mathrm{G}$ band position: $1605 \mathrm{~cm}^{-1}$ before treatment, $1595 \mathrm{~cm}^{-1}$ after treatment).

Electron microscopy studies were carried out to shed light on the morphology of the prepared N-doped sample. High resolution transmission electron microscopy (HRTEM) images exhibit typical wrinkled morphologies with a mixture of single and (a)

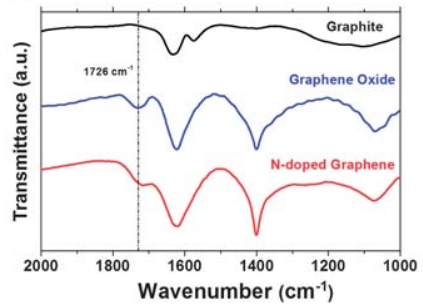

(b)

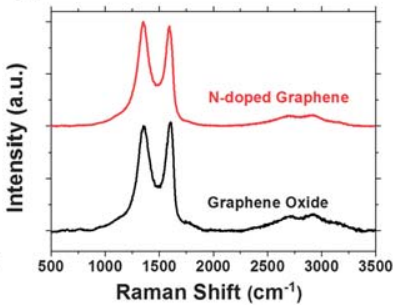

Fig. 2 (a) FTIR and (b) Raman spectra of graphite, graphene oxide and N-doped graphene. few-layer graphene nanosheets as shown in Fig. 3a. Inset in the image is an associated Selected Area Electron Diffraction (SAED) pattern. This shows discrete diffraction spots indicative of few or bi-layer material with high levels of graphitic crystallinity. Some variation of graphitic crystalline ordering was observed across different sample flakes (see ESI $\dagger$ ) with flakes displaying differing levels of crystalline disorder. This disorder is attributed to the presence of the functional groups previously discussed in the spectroscopic characterisation. Powder samples of N-doped graphene were analysed using Scanning Electron and Helium Ion Microscopy (SEM and HIM, respectively). The SEM image in Fig. 3c shows the bulk structure of the powder. This is shown in even greater detail in the HIM image in Fig. 3d, due to the higher resolution and greater depth of focus afforded by this tool. The layered structure of the bulk material is clearly observed. The oxidation of graphite during the production of the parent GO causes the graphitic layers to expand and exfoliate on oxidation. The nature of the gas phase plasma treatment is such that no reaggregation of the $\mathrm{N}$-doped graphene flakes occurs after reduction; resulting in a similar expanded layered structure in the resultant material.

The electrochemical properties of the $\mathrm{N}$-doped graphene material were investigated using Cyclic Voltammetry (CV) and Electrochemical Impedance Spectroscopy (EIS). The ferri/ ferrocyanide redox couple was employed to elucidate the electron transfer characteristics of the material in a three electrode set-up. It has previously been shown that pyrolytic carbon (PyC) films (grown by a non-catalysed CVD technique on $\mathrm{SiO}_{2}$ wafers) exhibit good conductivity but very sluggish electron transfer kinetics. ${ }^{24,25} \mathrm{~N}$-doped graphene (3 mg) was dispersed in DMF $(250 \mu \mathrm{l})$ and mildly sonicated to ensure a homogeneous dispersion. $30 \mu \mathrm{l}$ of this dispersion was dropped onto an asgrown PyC film and thoroughly dried at $80{ }^{\circ} \mathrm{C}$ for one hour. The graphene-coated PyC wafer was then employed as the working

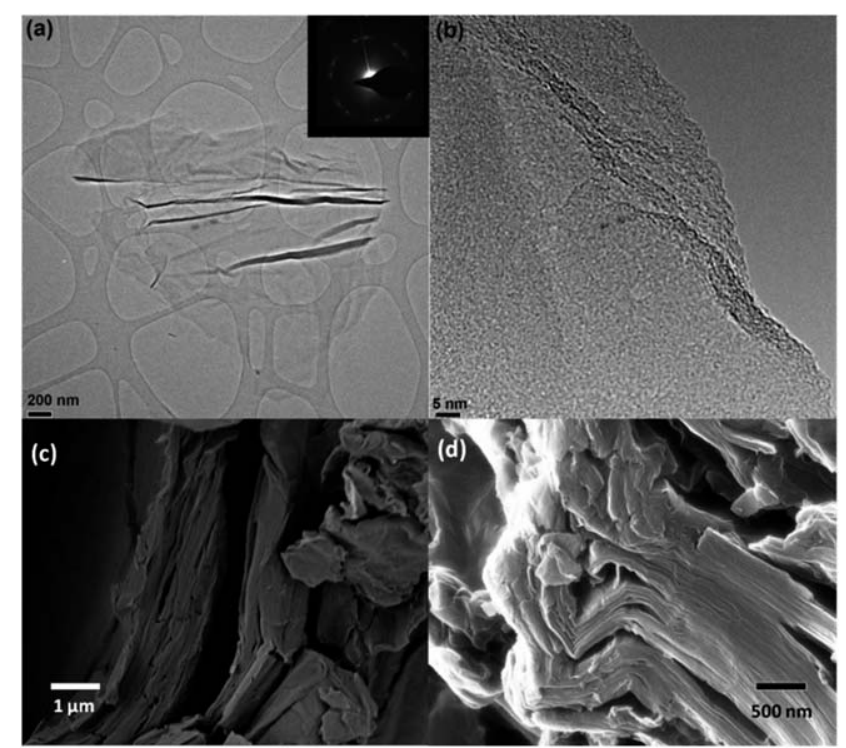

Fig. 3 HRTEM images of (a) N-doped graphene with SAED pattern and (b) edge image. (c) SEM image and (d) He-ion image of N-doped graphene powder. 
electrode using a home-made electrode assembly as previously described elsewhere. ${ }^{24,25}$

A solution of $1 \mathrm{mM}$ potassium ferricyanide and potassium ferrocyanide in $1 \mathrm{M} \mathrm{KCl}$ was prepared and thoroughly degassed by bubbling $\mathrm{N}_{2}$ gas through for 30 minutes. CV was carried out on the samples at a range of scan rates. Shown in Fig. 4a are cyclic voltammograms for PyC and $\mathrm{N}$-doped graphene at scan rates of $100 \mathrm{mV} \mathrm{s}^{-1}$. The increased current of the $\mathrm{N}$-doped graphene sample compared to the bare PyC substrate is likely due to increased electrochemical surface area resulting from the rougher surface of the $\mathrm{N}$-doped graphene decorating the otherwise flat PyC surface. As indicated in Fig. 4a, the peak separation, $\Delta E_{\mathrm{p}}$, for the $\mathrm{N}$-doped graphene is narrower than that for bare PyC; indicating faster electron transfer kinetics for the $\mathrm{N}$-doped graphene film. The homogeneous electron transfer rate constant, $k^{0}$, may be calculated using the Nicholson approach; ${ }^{26}$ which is given a more rigorous treatment elsewhere. ${ }^{27}$ Calculations for $k^{0}$ using $\Delta E_{\mathrm{p}}$ data for scan rates of 100 $\mathrm{mV} \mathrm{s}^{-1}$ yielded values of $k^{0}=1.28 \times 10^{-3} \mathrm{~cm} \mathrm{~s}^{-1}$ for PyC and $k^{0}$ $=5.17 \times 10^{-3} \mathrm{~cm} \mathrm{~s}^{-1}$ for $\mathrm{N}$-doped graphene. EIS analysis of the same materials indicates a reduced resistance to charge transfer for the N-doped graphene compared to the as-grown PyC. This is illustrated by the smaller semi-circular region in the Nyquist plots in Fig. $4 \mathrm{~b}$.

Preliminary evaluation of the catalytic performance of the Ndoped material for Oxygen Reduction Reaction (ORR) was carried out by drop-casting the material on to a glassy carbon (GC) disc and employing this as the working electrode in an electrochemical cell with $1 \mathrm{M} \mathrm{NaOH}$ electrolyte. The electrolyte was thoroughly degassed with bubbled $\mathrm{N}_{2}$ and $\mathrm{CV}$ was performed. The electrolyte was then saturated with $\mathrm{O}_{2}$ and the measurement repeated.

A clear redox peak is introduced in the corresponding voltammograms (Fig. 5a) which is associated with the reduction of molecular oxygen. Rotating Disc and Rotating Ring Disc Electrode (RDE and RRDE, respectively) measurements were carried out to gain further insight into the mechanism of the reduction reaction at the $\mathrm{N}$-doped graphene electrode. Linear Sweep Voltammetry (LSV) was carried out at a scan rate of $20 \mathrm{mV} \mathrm{s}^{-1}$ on the $\mathrm{N}$-doped graphene disk while the Pt ring was held at a constant potential of $0.45 \mathrm{~V}$. Fig. 5b shows the voltammograms corresponding to a RRDE rotation speed of $2000 \mathrm{rpm}$ with the current owing to oxygen reduction shown in black and the corresponding
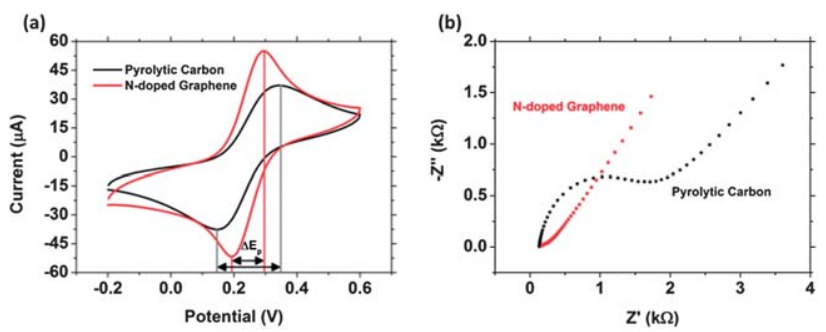

Fig. 4 (a) Cyclic voltammograms of as-grown PyC (black) and N-doped graphene on PyC (red), peak separation $\left(\Delta E_{\mathrm{p}}\right)$ is marked. The scan rate is $100 \mathrm{mV} \mathrm{s}^{-1}$. (b) Corresponding Nyquist plots for the two materials.
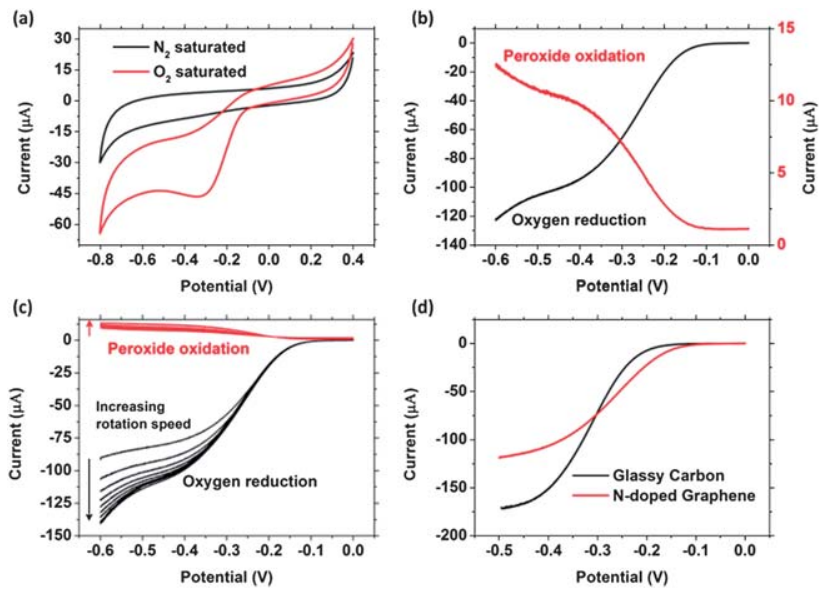

Fig. 5 (a) Cyclic voltammograms for $\mathrm{N}$-doped graphene in $\mathrm{N}_{2}$ saturated (black) and $\mathrm{O}_{2}$ saturated (red) $1 \mathrm{M} \mathrm{NaOH}$. (b) LSVs for RDE measurement of $\mathrm{N}$-doped graphene showing currents owing to oxygen reduction (black) and peroxide oxidation (red); rotation speed of $2000 \mathrm{rpm}$. (c) A series of LSVs for the same material at various rotation speeds, ORR and peroxide currents are included. (d) LSVs for RDE measurements of GC (black) and N-doped graphene (red).

current owing to hydrogen peroxide oxidation in red. The values on the left $y$-axis correspond to the ORR current, whilst the values on the right $y$-axis correspond to oxidation of hydrogen peroxide. This indicates that the oxygen reduction proceeds via an energetically favourable four electron pathway. ${ }^{28}$ Fig. $5 \mathrm{c}$ shows a series of LSVs for the N-doped graphene RRDE at different rotation speeds; starting at $500 \mathrm{rpm}$ with $500 \mathrm{rpm}$ increments between each scan. Included are the currents corresponding to the peroxide oxidation. The performance of the N-doped graphene film was compared to that of bare glassy carbon to eliminate any substrate contributions. Shown in Fig. 5d are RDE LSVs for the two electrode types. The GC displays a higher steady state current than the $\mathrm{N}$-doped graphene, but this is easily explained by the greater conductivity in the GC material. Of particular note is the lower onset potential of the ORR peak for the N-doped graphene compared to the GC. This difference of $\sim 50 \mathrm{mV}$ indicates that there exists a potential catalytic effect of the $\mathrm{N}$-doped graphene on the reduction of oxygen.

\section{Conclusions}

To summarise, we have developed a robust process for the simultaneous reduction and N-doping of GO, as confirmed by rigorous spectroscopic characterisation. This dry process is scalable and produces $\mathrm{N}$-doped graphene flakes with a high degree of exfoliation. Initial studies indicate that the material produced is a promising candidate for metal free ORR catalysis. The facile synthesis of this material could be of great benefit for the production of fuel cells and other emerging energy technologies.

\section{Acknowledgements}

This work was supported by the SFI under Contracts no. 08/CE/ I1432, PI_10/IN.1/I3030 and the EU under FP7-2010 GC-ELECTROCHEMICAL-STORAGE (Electrograph Co no. 266391). The 
authors thank Dr Alan Bell of the Advanced Microcopy Laboratory (AML) of CRANN for facilitating Helium-Ion microscopy.

\section{Notes and references}

1 H. Wang, T. Maiyalagan and X. Wang, ACS Catal., 2012, 2, 781-794.

2 Y. Shao, S. Zhang, M. H. Engelhard, G. Li, G. Shao, Y. Wang, J. Liu, I. A. Aksay and Y. Lin, J. Mater. Chem., 2010, 20, 74917496.

3 L. Qu, Y. Liu, J.-B. Baek and L. Dai, ACS Nano, 2010, 4, 13211326.

4 A. L. M. Reddy, A. Srivastava, S. R. Gowda, H. Gullapalli, M. Dubey and P. M. Ajayan, ACS Nano, 2010, 4, 6337-6342.

5 Y. Wang, Y. Shao, D. W. Matson, J. Li and Y. Lin, ACS Nano, 2010, 4, 1790-1798.

6 I. K. Moon, J. Lee, R. S. Ruoff and H. Lee, Nat. Commun., 2010, 1, 73.

7 S. Pei and H.-M. Cheng, Carbon, 2012, 50, 3210-3228.

8 Z. Wei, D. Wang, S. Kim, S.-Y. Kim, Y. Hu, M. K. Yakes, A. R. Laracuente, Z. Dai, S. R. Marder, C. Berger, W. P. King, W. A. de Heer, P. E. Sheehan and E. Riedo, Science, 2010, 328, 1373-1376.

9 D. Long, W. Li, L. Ling, J. Miyawaki, I. Mochida and S.-H. Yoon, Langmuir, 2010, 26, 16096-16102.

10 X. Li, H. Wang, J. T. Robinson, H. Sanchez, G. Diankov and H. Dai, J. Am. Chem. Soc., 2009, 131, 15939-15944.

11 N. Li, Z. Wang, K. Zhao, Z. Shi, Z. Gu and S. Xu, Carbon, 2010, 48, 255-259.

12 D. Wei, Y. Liu, Y. Wang, H. Zhang, L. Huang and G. Yu, Nano Lett., 2009, 9, 1752-1758.

13 S. Maldonado and K. J. Stevenson, J. Phys. Chem. B, 2005, 109, 4707-4716.
14 Y. J. Cho, H. S. Kim, H. Im, Y. Myung, G. B. Jung, C. W. Lee, J. Park, M.-H. Park, J. Cho and H. S. Kang, J. Phys. Chem. C, 2011, 115, 9451-9457.

15 N. P. Subramanian, X. Li, V. Nallathambi, S. P. Kumaraguru, H. Colon-Mercado, G. Wu, J.-W. Lee and B. N. Popov, J. Power Sources, 2009, 188, 38-44.

16 R. Imran Jafri, N. Rajalakshmi and S. Ramaprabhu, J. Mater. Chem., 2010, 20, 7114-7117.

17 N. A. Kumar, H.-J. Choi, Y. R. Shin, D. W. Chang, L. Dai and J.-B. Baek, ACS Nano, 2012, 6, 1715-1723.

18 X. Mei and J. Ouyang, Carbon, 2011, 49, 5389-5397.

19 S. Stankovich, D. A. Dikin, R. D. Piner, K. A. Kohlhaas, A. Kleinhammes, Y. Jia, Y. Wu, S. T. Nguyen and R. S. Ruoff, Carbon, 2007, 45, 1558-1565.

20 H. L. Poh, F. Šaněk, A. Ambrosi, G. Zhao, Z. Sofer and M. Pumera, Nanoscale, 2012, 4, 3515-3522.

21 O. C. Compton, D. A. Dikin, K. W. Putz, L. C. Brinson and S. T. Nguyen, Adv. Mater., 2010, 22, 892-896.

22 D. Sun, X. Yan, J. Lang and Q. Xue, J. Power Sources, 2013, 222, 52-58.

23 Y.-C. Lin, C.-Y. Lin and P.-W. Chiu, Appl. Phys. Lett., 2010, 96, 133110-133113.

24 G. P. Keeley, N. McEvoy, S. Kumar, N. Peltekis, M. Mausser and G. S. Duesberg, Electrochem. Commun., 2010, 12, 10341036.

25 N. McEvoy, N. Peltekis, S. Kumar, E. Rezvani, H. Nolan, G. P. Keeley, W. J. Blau and G. S. Duesberg, Carbon, 2012, 50, 1216-1226.

26 R. S. Nicholson, Anal. Chem., 1965, 37, 1351-1355.

27 M. Lyons and G. Keeley, Sensors, 2006, 6, 1791-1826.

28 Y. Liang, H. Wang, P. Diao, W. Chang, G. Hong, Y. Li, M. Gong, L. Xie, J. Zhou, J. Wang, T. Z. Regier, F. Wei and H. Dai, J. Am. Chem. Soc., 2012, 134, 15849-15857. 